$\underline{\text { Review Article }}$

\title{
UTILIZATION OF EOSIN DYE AS AN ION PAIRING AGENT FOR DETERMINATION OF PHARMACEUTICALS: A BRIEF REVIEW
}

\author{
HABIBUR RAHMAN \\ Department of General Studies, Jubail Industrial College, P. 0. Box 10099, Jubail Industrial City 31961, Saudi Arabia \\ Email: habibur_r@jic.edu.sa
}

Received: 16 Jun 2017 Revised and Accepted: 02 Nov 2017

\begin{abstract}
Globally, dyes are widely used in the pharmaceutical, food, textile, cosmetics, plastics, leather, paint, ink and paper industries. Eosin is an acidic orange-pink dye and has very strong staining properties. Haematoxylin and eosin $\mathrm{Y}$ ( $\mathrm{H}$ and E) combination is the most common staining and primary diagnostic technique in histo-pathological laboratories. This review mainly discussed the utility of eosin dye in quality control laboratories as an ion pairing agent for drug analysis. Eosin Y is one the most common ion pairing agent and its mono and di anionic forms are capable of interacting with many drug molecules to form colored/fluorescent binary or ternary complexes that can be analyzed with or without extraction by spectrofluorimetry and/or spectrophotometry. Quenching fluorescence and advantages of spectrofluorimetry over spectrophotometry were also discussed. Fluorescence detection greatly enhances the sensitivity and providing a sensitive and relatively inexpensive instrumental method of analysis using eosin Y for various important drugs in pure, commercial dosage forms and biological fluids.
\end{abstract}

Keywords: Eosin Y, Ion pairing agent, Binary and ternary complexes, Commercial dosage forms

(C) 2017 The Authors. Published by Innovare Academic Sciences Pvt Ltd. This is an open access article under the CC BY license (http://creativecommons.org/licenses/by/4.0/) DOI: http://dx.doi.org/10.22159/ijpps.2017v9i12.21220

\section{INTRODUCTION}

Dyes are natural or synthetic colored organic substances which have the affinity to impart color to various substrates by absorbing into the substrate. In general, the dye molecules are chemically bonded to the surface and become a part of the material on which it is applied. Dyes are widely used in the pharmaceutical, food, textile, cosmetics, plastics, leather, paint, ink and paper industries<smiles>[Y]CCOc1ccccc1-c1c2cc(Br)c(=O)c(Br)c-2oc2c(Br)c(O[N+]([O-])OCC)c(Br)cc12</smiles>

[1]. A class of dye called xanthene is classified into three subgroups. One of the subgroups is called fluorone dyes that include fluorescein, erythrosine and rhodamine.

Eosin dyes are bromine derivative of fluorescein which has two very closely related dyes commonly known as Eosin yellowish (Eosin Y) and Eosin bluish (Eosin B) as shown in fig. 1. Eosin $\mathrm{Y}$ is a tetrabromo derivative whereas eosin $\mathrm{B}$ is a dibromo dinitro derivative of fluorescein.

Fig. 1: Structure of eosins

Eosin Y is chemically known as disodium 2-(2, 4, 5, 7-tetrabromo-6oxido-3-oxo-3H-xanthen-9-yl) benzoate have a molecular formula $\left(\mathrm{C}_{20} \mathrm{H}_{6} \mathrm{Br}_{4} \mathrm{Na}_{2} \mathrm{O}_{5}\right)$ and molar mass 691.85. Fluorescein in the eosin $\mathrm{Y}$ molecule called fluorochrome exists in two forms; one is the more stable quinoid form which is colored and fluorescent while the other one is lactone form which is colorless and non-fluorescent presented as shown A and B respectively, in fig. 2 [2]. Disodium salt of eosin Y can be readily converted into free acid in which the free carboxylic acid group (quinoid form) exists in equilibrium with its lactone form. In a weakly acidic medium, ionization of eosin $\mathrm{Y}$ may take place either by dissociation of hydroxyl or carboxylic group.

It was reported that hydroxyl group dissociates easily compare to the carboxylic acid group. In solution, eosin $\mathrm{Y}$ can exist in three different forms as neutral $\left(\mathrm{H}_{2} \mathrm{R}\right)$, monoanionic (HR-) and di anionic $\left(\mathrm{R}^{2-}\right)$ forms, where $\mathrm{R}$ denotes the anionic part of the eosin $\mathrm{Y}$ as explained in fig. 3 [3-4]. The ionization constants ( $\mathrm{pKa} 1$ and $\mathrm{pKa} 2$ ) of eosin Y were reported as 2.9 and 4.5 [5].
Eosin $\mathrm{Y}$ is a Biological Stain Commission certified dye. The HandE stains the most common and primary diagnostic technique in histopathological laboratories to stain cells and cytoplasm, collagen and muscle fibers. $\mathrm{H}$ and $\mathrm{E}$ staining method was also used in structure determination of grasshopper and mammalian testis as well as supporting structure determination of destruction of dental tissues [6]. Eosin was utilized as a fluorescent indicator in acid-base titration for the determination of vitamins $\left(\mathrm{B}_{1}\right.$ and $\left.\mathrm{B}_{2}\right)$ in food [7] Due to its strong staining properties, it has been used in various industrial applications such as color filter, liquid crystal display, paper inks, photographic materials, laundry detergent, cosmetics, pencil lead, pigments, varnish and textiles. It is also used in medicine as an important radioactive tracer.

Literature surveys reported that the mono or di anionic forms of eosin $\mathrm{Y}$ are capable of interacting with a cationic form of the drug molecules by the electrostatic interaction and hydrophobic forces and forms either binary complex with drug molecule or a ternary complex with drug molecule and a metal [8]. 
<smiles>O=C(O)c1ccccc1-c1c2cc(Br)c(=O)c(Br)c-2oc2c(Br)c(O)c(Br)cc12</smiles><smiles></smiles>

Eosin Y

Fig. 2: Different forms of fluorochrome (Eosin Y)

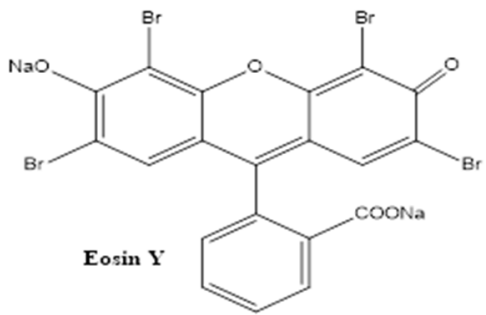

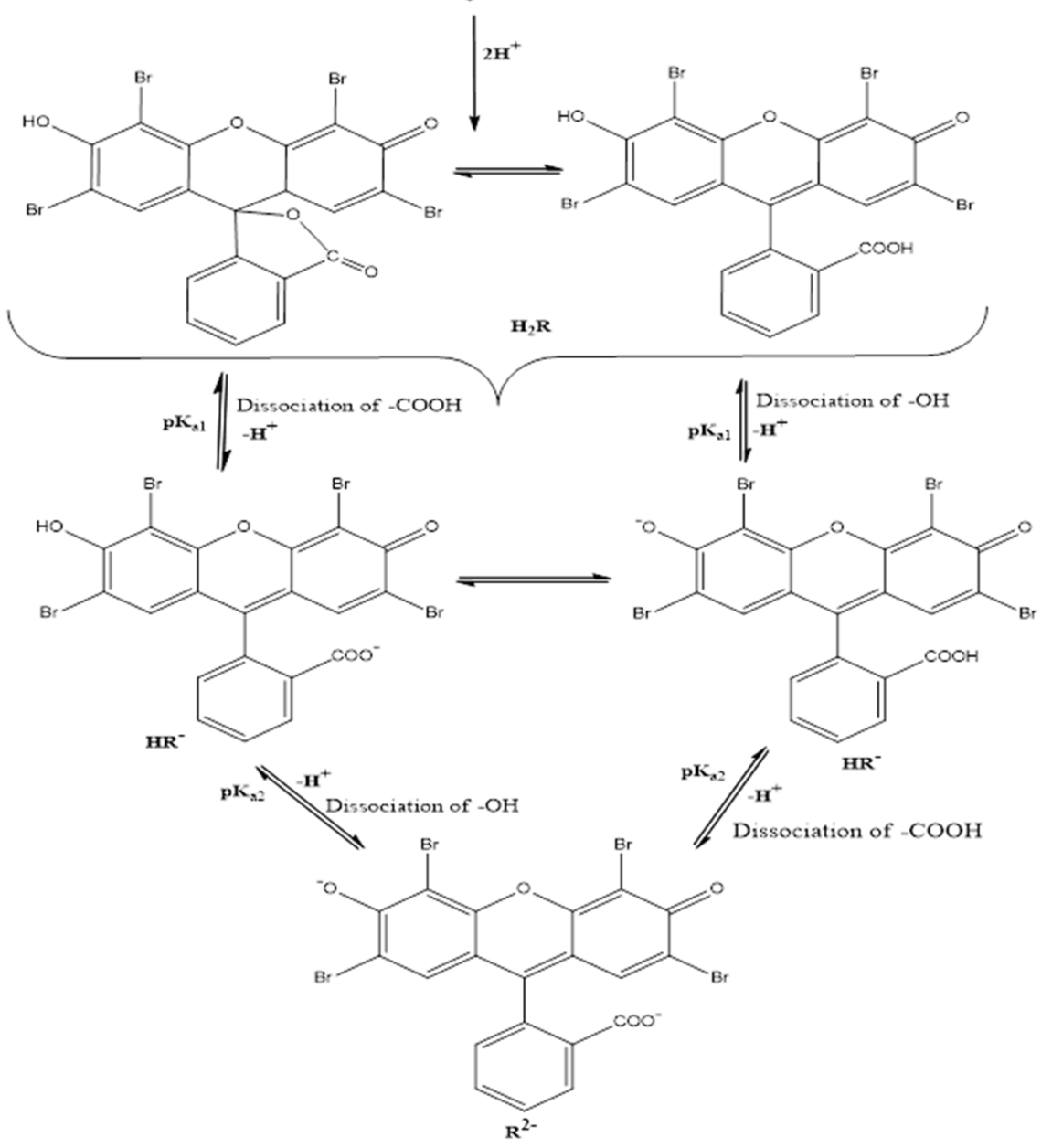

Fig. 3: Ionization of eosin $Y$ and its three different forms $\left(H_{2} R\right.$, HRand $\left.R^{2-}\right)$ [3-4]

In recent years, eosin was used as photoredox catalyst in organic synthesis [9], Carbon-Carbon and Carbon-Phosphorous bond formation [10]. It was utilized in the cleavage of C-C double bond of styrene [11], binding and estimation of protein assay [12-14], antimalarial agent for drug resistant Plasmodium falciparum [15], activating agent in teeth whitening composition [16], tracer in groundwater studies [17], complexing agent in ternary ion- association complex nanoparticles [18-19], sensing material in preparation of eosin Y film modified glassy carbon electrode [20], photosensitizer in dye sensitized solar cells and topical agents for treatment of diaper dermatitis and interfering agent in measurement of serum vancomycin [21-23]. Indirect determination of histamine, an important compound in various physiological processes in humans was determined in fish samples, dairy products 
and alcoholic beverages complexation with Fe (III) and eosin Y [24]. Catalyst-free activation of peroxides through photoexcited electron under visible LED light irradiation was also performed using eosin Y as a model dye [25]. A highly selective and sensitive resonance light scattering detection approach was developed for the synchronous analysis of anthelmintics after reaction with eosin $Y$ to form ionassociation complexes and separated by high-performance liquid chromatography [26]. An environmentally useful method was developed to detect sodium dodecyl sulphate using eosin $Y$ and polyethyleneimine complex [27].

\section{Applicability of eosin $\mathrm{Y}$ in drug analysis}

Several commercially available xanthene dyes have been successfully used in the analysis of many pharmaceutical compounds. Eosin $\mathrm{Y}$ is an acidic dye belongs to the xanthene group of dyes that has been widely used for the determination of several basic drugs through the formation of colored or fluorescent ion association complex using spectrophotometric and/or spectrofluorimetric methods.

\section{Formation of binary and ternary complexes}

Binary ion association complexes formed between eosin and the drug molecules by electrostatic interaction. It was reported that the formation of binary complex increases the sensitivity of determination. It may decrease the fluorescence intensity of the native fluorescence of the eosin [28] without any permanent change in the molecule. The stability and fluorescence capacity of the ion association complexes were studied by using various acidic buffer solutions and dispersing agents. It was reported that $\mathrm{pH}$ is the critical factor which plays an important role on ionization of eosin Y. It was found that the fluorescence capacity of the ion association complex formed with eosin $\mathrm{Y}$ was increased up to certain $\mathrm{pH}$ values and then a decrease in the fluorescence intensity was occurred [2931]. Dispersing agents also affect the stability of the complexes and prevents precipitation of the ion association complexes [32-33].

Ternary complex categorized into two major types, the one is mixed ligand complex and the other is an ion-association complex. Both types of ternary complex formation improve not only the sensitivity but also the selectivity as well. The sensitivity of the ternary complex can also enhance by the addition of surfactant that dissolves the ternary complex and measured directly without involving extraction process. In general, a ternary complex has formula $L_{n} M_{x} S_{y}$, where $L$ stands for main ligand i.e. cited drug, $\mathrm{M}$ is the metal and $\mathrm{S}$ is the eosin Y, respectively.

The mechanism of ternary complex formation includes coordinate bond formation between metal ion and the cited drug molecule through atoms carrying lone pairs of electrons, and subsequently, a ternary complex is formed by interaction with eosin Y. The major advantage of ternary complex formation that it often has higher values of molar absorptivity and therefore improves the sensitivity of the method. Metal chelates formation also promotes the fluorescence and forms a ternary complex with eosin Y which can be utilized in the indirect determination of metals using atomic absorption spectrophotometric technique. These complexes are extractable with organic solvents such as chloroform and methylene chloride and have been widely utilized in the analysis of several pharmaceuticals. Eosin Y was used for determination of various pharmaceutical compounds using spectrophotometry and/or spectrofluorimetry through the formation of ternary complexes. However, the metal-drug and metal-eosin binary systems cannot be extracted in the same manner.

\section{Quenching fluorescence}

The term quenching refers to a process that decreases the fluorescence intensity of a given substance. It occurs during excited state lifetime and results in various types of molecular interactions. Collisional or dynamic quenching and static quenching are the two main types of quenching process. Both types of quenching require interaction between the fluorophore and quencher molecule. In dynamic quenching, the quencher molecule must diffuse to the fluorophore during excited state lifetime and upon contact, the fluorophore returns to the ground state without photon emission.
Static quenching occurs when a fluorophore and quencher create a non-fluorescent complex before excitation of fluorophore [34]. Molecular oxygen, iodide, bromide ions and acrylamide are common quenchers for almost all dyes [35-38].

In addition to the above, apparent quenching can also occur due to the optical properties of the samples which are not much more useful. This trivial type of quenching occurs due to high optical densities or turbidity and can easily be controlled.

A separate type of quenching known as Fluorescence Resonance Energy Transfer (FRET) in which the intensity of the donor decreases and transfer to an acceptor molecule. The acceptor can be fluorescent or non-fluorescent. In both cases, the fluorescence intensity of the initially excited molecule is decreased. This type of quenching is often referred to as a donor-acceptor pair. It is mediated by the emission of a photon and it does not even require that the acceptor chromophore be fluorescent. During photosynthesis, a light antenna pigment uses resonance energy transfer to donate the collected energy to the photosynthetic reaction centre. This technique has led to qualitative and quantitative improvements, including increased spatial resolution and sensitivity. FRET is commonly used to measure distances within or between molecules in protein studies [39]. At present, FRET was used for measuring the structure [40-42], conformational changes [43] and interactions between molecules [44-45].

\section{Applications of spectrofluorimetry in drug analysis using eosin}

Spectrofluorimetry or fluorescence spectroscopy is a type of molecular emission spectroscopy and an extremely sensitive analytical method which has been widely applied for the determination of a variety of pharmaceutical compounds. It measures the fluorescence intensity which allows sensitive and selective quantitation of certain compounds which exhibit the fluorescence. This method involves measurement of enhanced or quenched fluorescent signals. It has been applied in three different processes: fluorescence, phosphorescence and chemiluminescence. Fluorescence is the most common luminescence process used in the pharmaceutical analysis. It involves photoexcitation process which occurs by absorption of various types of radiant energy such as UVvisible light and emission process in which emission radiant energy from an excited electronic state takes place as fluorescence within $10^{-6}$ to $10^{-9}$ seconds. Mostly fluorescent molecules are aromatic in nature. However, substituents such as- $-\mathrm{NH}_{2}-\mathrm{OH},-\mathrm{F},-\mathrm{OCH}_{3},-\mathrm{NHCH}_{3}$ and $\mathrm{N}\left(\mathrm{CH}_{3}\right)_{2}$ groups, often enhances fluorescence while electron withdrawing groups containing halogen such as- $\mathrm{Cl},-\mathrm{Br},-\mathrm{I}$, and$\mathrm{NHCOCH}_{3},-\mathrm{NO}_{2}$ or- $\mathrm{COOH}$ decrease or quench completely the fluorescence. Presence of dissolved oxygen, changes in buffer solutions of different $\mathrm{pH}$ values and solvent polarity also exhibit marked effect on the fluorescence of compounds.

This technique is important because of the fact that the intensity of light emitted by a fluorescent compound depends upon the concentration of that compound and hence, the measurement of fluorescence intensity permits the quantitative determination of trace contaminants of many inorganic species in the environment, industries and bodies. Fluorescence spectroscopy applied to drug analysis provides analytical methodologies with improved sensitivity, selectivity and range. It is one of the most important analytical methods in the field of chemistry, biology and chemical engineering. It is extensively used in nuclear research for the determination of uranium salts. It is also a method of the choice for the determination of many pharmaceutical compounds, plant pigments, hormones, food products, steroids and vitamins in formulations and biological fluids [46].

Taking the advantage of fluorescent properties of eosin Y, several researchers utilized eosin as a fluorescence quenching agent and analyses a variety of organic compounds. Eosin commonly used as an acidic red stain for highlighting cytoplasm material in samples. It forms either binary or ternary complex with pharmaceuticals and measured either directly without extraction or indirectly by extraction in an organic solvent. Many pharmaceutical compounds were analyzed using spectrofluorimetric technique involving eosin as a reactant. It was used in the analysis of various illicit drug 
samples encountered in small amounts [47]. Coumarins were determined in a well-known traditional Indian drug 'Shankhpushpi' [48], determination of scopoletin and mangiferin curcumin and testosterone in biological fluids [49-51].

The spectrofluorimetric method is less tedious and less cumbersome compared to chromatographic methods which require long run time and suffer from tedious operation procedures. Moreover, the high sensitivity and specificity offered by this method are also higher and the drug compounds can be analyzed up to nano levels. As data are shown in table 1, these methods were widely used in quantitation of many pharmaceuticals compounds using eosin $Y$ due to its simplicity, low cost, high sensitivity and wide concentration range [52-68].

\section{Applications of spectrophotometry in drug analysis using eosin}

Spectrophotometry is a type of absorption spectroscopy. It involves measuring the amount of ultraviolet or visible light absorbed by a substance in solution. It is one of the most frequently used methods for the quantitation of pharmaceuticals due to its low cost, ease of operation and simplicity. It has been regarded as one of the most suitable and economical methods in research laboratories, hospitals and pharmaceutical industries. Visible region spectrophotometric methods found suitable for single component analysis [69, 70]. However, these methods were found not suitable for the multicomponent mixture. The instrumental development of ultraviolet region absorption spectrophotometry was reviewed and applied to solve multicomponent pharmaceutical mixture [71]. Eosin $\mathrm{Y}$ formed a binary or ternary colored nonfluorescent complexes were analyzed by spectrophotometry. A number of pharmaceutical compounds were analyzed using eosin $\mathrm{Y}$ as an ion pairing agent shown in table 2 [72-93].

Comparison of spectrofluorimetric and spectrophotometric methods of analysis using eosin y

It has been reported that eosin $\mathrm{Y}$ is a common dye used for determination of various pharmaceutical compounds either spectrophotometry alone or spectrophotometry and spectrofluorimetry by the formation of a binary or ternary complex. Fig. 4 shows a schematic explanation that how the ion association complex formed with eosin $\mathrm{Y}$ and analyzed by spectrophotometry and spectrofluorimetry.

Table 1: Spectrofluorimetric determination of drugs using eosin $Y$

\begin{tabular}{|c|c|c|c|c|c|c|c|c|}
\hline \multirow[t]{2}{*}{ Drug } & \multirow{2}{*}{$\begin{array}{l}\text { Complex } \\
\text { type }\end{array}$} & \multirow{2}{*}{$\begin{array}{l}\lambda_{\text {em }} \\
(\mathrm{nm})\end{array}$} & \multirow{2}{*}{$\begin{array}{l}\lambda_{\text {ex }} \\
(\mathrm{nm})\end{array}$} & \multirow{2}{*}{$\begin{array}{l}\text { Range } \\
\mu \mathrm{g} / \mathrm{ml}\end{array}$} & \multirow{2}{*}{$\begin{array}{l}\text { LODa }^{a} \\
\mu \mathrm{g} / \mathrm{ml}\end{array}$} & \multirow{2}{*}{$\begin{array}{l}\mathrm{LOQ}^{\mathrm{b}} \\
\mu \mathrm{g} / \mathrm{ml}\end{array}$} & \multirow[t]{2}{*}{ Application } & \multirow[t]{2}{*}{ Reference } \\
\hline & & & & & & & & \\
\hline Olanzapine & Ternary & 547 & 323 & $0.05-1.0$ & 0.0018 & 0.006 & $\begin{array}{l}\text { Pharmaceutical preparations and } \\
\text { human plasma }\end{array}$ & 52 \\
\hline Fluphenazine & Ternary & 547 & 323 & $0.10-1.0$ & 0.0012 & 0.004 & $\begin{array}{l}\text { Pharmaceutical preparations and } \\
\text { human plasma }\end{array}$ & 52 \\
\hline Risperidone & Ternary & 555 & 260 & $0.5-7$ & 0.015 & 0.050 & Bulk and tablets & 53 \\
\hline Labetalol & Ternary & 452 & 317 & $0.5-4$ & 0.08 & 0.23 & $\begin{array}{l}\text { Pharmaceutical preparations and } \\
\text { urine }\end{array}$ & 56 \\
\hline Doxepin $\mathrm{HCl}$ & Binary & 567 & 464 & $0.1-8$ & 0.00295 & 0.00975 & Commercial dosage forms & 54 \\
\hline Betaxolol & Binary & 545 & 301.5 & $0.1-2.5$ & 0.028 & 0.086 & dosage forms & 55 \\
\hline Carvedilol & Binary & 545 & 301.5 & $0.1-2.5$ & 0.024 & 0.071 & dosage forms & 55 \\
\hline Labetalol & Binary & 545 & 301.5 & $0.1-2.5$ & 0.057 & 0.172 & dosage forms & 55 \\
\hline Nebivolol & Binary & 545 & 301.5 & $0.1-2.5$ & 0.046 & 0.14 & dosage forms & 55 \\
\hline Propranolol & Binary & 545 & 301.5 & $0.1-2.5$ & 0.016 & 0.05 & dosage forms & 55 \\
\hline Labetalol & Binary & 432 & 312 & $1.25-30$ & 0.24 & 0.73 & $\begin{array}{l}\text { Pharmaceutical preparations and } \\
\text { urine }\end{array}$ & 56 \\
\hline Chloroquine & Binary & 372 & 318 & $0.5-5$ & - & - & Dosage forms & 57 \\
\hline Amodiaquine & Binary & 368 & 318 & $0.5-8$ & - & - & Dosage forms & 57 \\
\hline Primaquine & Binary & 450 & 368 & $0.1-5$ & - & - & Dosage forms & 57 \\
\hline Clindamycin $\mathrm{HCl}$ & Binary & 555 & 482 & $0.2-2$ & 0.13 & 0.18 & Pure and dosage forms & 58 \\
\hline Almotriptan malate & Binary & 542.8 & 301.3 & $0.07-1.0$ & 0.019 & 0.059 & Pure and dosage forms & 59 \\
\hline Rizatriptan benzoate & Binary & 542.8 & 301.3 & $0.20-1.0$ & 0.041 & 0.125 & Pure and dosage forms & 59 \\
\hline Sumatriptan succinate & Binary & 542.8 & 301.3 & $0.20-1.0$ & 0.055 & 0.168 & Pure and dosage forms & 59 \\
\hline Zolmitriptan & Binary & 542.8 & 301.3 & $0.10-1.0$ & 0.032 & 0.096 & Pure and dosage forms & 59 \\
\hline Losartan & Binary & 546 & 310 & $0.8-8$ & 0.203 & 0.617 & Tablets & 60 \\
\hline Irbesartan & Binary & 546 & 310 & $0.8-7$ & 0.110 & 0.335 & Tablets & 60 \\
\hline Telmisartan & Binary & 546 & 310 & $0.9-4$ & 0.112 & 0.340 & Tablets & 60 \\
\hline Valsartan & Binary & 546 & 310 & $1-8$ & 0.132 & 0.399 & Tablets & 60 \\
\hline Amitriptyline $\mathrm{HCl}$ & Binary & 550 & 310 & $0.08-2$ & 0.017 & 0.056 & Pharmaceutical preparations & 61 \\
\hline Clomipramine $\mathrm{HCl}$ & Binary & 550 & 310 & $0.06-2$ & 0.015 & 0.049 & Pharmaceutical preparations & 61 \\
\hline Citalopram $\mathrm{HBr}$ & Binary & 554 & 259 & $2-26$ & 0.121 & - & Dosage forms & 62 \\
\hline Fluoxetine $\mathrm{HCl}$ & Binary & 545 & 301 & $0.2-2.4$ & 0.066 & 0.036 & Pharmaceutical formulations & 63 \\
\hline Paroxetine $\mathrm{HCl}$ & Binary & 545 & 301 & $0.1-2.4$ & 0.20 & 0.10 & Pharmaceutical formulations & 63 \\
\hline Ethionamide & Binary & 536 & 337 & $1-8$ & 0.08 & - & $\begin{array}{l}\text { Pharmaceutical preparations and } \\
\text { biological fluids }\end{array}$ & 64 \\
\hline Thioridazine & Ternary & 517 & 462 & $0.5-3$ & - & - & Dosage forms & 65 \\
\hline Flupentixol & Ternary & 517 & 462 & $0.5-3$ & - & - & Dosage forms & 65 \\
\hline Sunitinib malate & Binary & 800 & 350 & $0.08-5$ & 0.041 & 0.85 & $\begin{array}{l}\text { Bulk and pharmaceutical } \\
\text { preparations }\end{array}$ & 66 \\
\hline Ebastine & Binary & 553 & 457 & $0.1-1.0$ & 0.021 & 0.042 & Pharmaceutical preparations & 67 \\
\hline $\begin{array}{l}\text { Prochlorperazinedimal } \\
\text { eate }\end{array}$ & Binary & 450 & 318 & $1-10$ & - & - & Pharmaceutical preparations & 68 \\
\hline $\begin{array}{l}\text { Thiethylperazinedihyd } \\
\text { rochloride }\end{array}$ & Binary & 460 & 318 & $1-10$ & - & - & Pharmaceutical preparations & 68 \\
\hline $\begin{array}{l}\text { Trifloperazinedihydroc } \\
\text { hloride }\end{array}$ & Binary & 465 & 318 & $1-10$ & - & - & Pharmaceutical preparations & 68 \\
\hline
\end{tabular}

${ }^{\mathrm{a}}$ Limit of detection, ${ }^{\mathrm{b}}$ Limit of quantitation. 
Table 2: Spectrophotometric determination of drugs using eosin $Y$

\begin{tabular}{|c|c|c|c|c|c|c|c|}
\hline Drug & $\begin{array}{l}\text { Complex } \\
\text { type }\end{array}$ & $\begin{array}{l}\lambda_{\max } \\
(\mathrm{nm})\end{array}$ & $\begin{array}{l}\text { Range } \\
\mu \mathrm{g} / \mathrm{ml}\end{array}$ & $\begin{array}{ll}\mathrm{LOD} \\
\mu \mathrm{g} / \mathrm{ml}\end{array}$ & $\begin{array}{l}\mathrm{LOQ} \\
\mu \mathrm{g} / \mathrm{ml}\end{array}$ & Application & Reference \\
\hline Tizanidine & Binary & 545 & $0.5-8$ & 0.1 & 0.26 & Dosage forms & 72 \\
\hline Orphenadrine & Binary & 542 & $1-12$ & 0.3 & 0.95 & Dosage forms & 72 \\
\hline $\begin{array}{l}\text { Clemastine hydrogen } \\
\text { fumarate }\end{array}$ & Binary & 552 & $1.25-11.25$ & 0.72 & 2.39 & Dosage forms & 73 \\
\hline Desloratadine & Binary & 549 & $0.31-2.81$ & 0.9 & 3 & Dosage forms & 73 \\
\hline Losartan potassium & Binary & 540 & $2.5-20$ & 0.82 & 2.73 & Dosage forms & 73 \\
\hline Moxepril HCl & Binary & 540 & $1.25-15$ & 0.75 & 2.51 & Dosage forms & 73 \\
\hline Bezafibrate & Ternary & 546 & $0.06-3$ & 0.00915 & 0.0277 & Pharmaceutical products & 74 \\
\hline Amlodipine & Binary & 549 & $5-60$ & 1.8 & 6.0 & Bulk powder and pharmaceutical formulations & 74 \\
\hline Nicardipine & Binary & 549 & $5-60$ & 1.2 & 3.6 & Bulk powder and pharmaceutical formulations & 74 \\
\hline Terbutaline sulphate & Binary & 545 & $0.5-10$ & 0.030 & 0.103 & Pharmaceutical formulations & 75 \\
\hline $\begin{array}{l}\text { Tetracycline } \\
\text { hydrochloride }\end{array}$ & Binary & 545 & $5-45$ & 0.613 & 2.00 & Pharmaceutical formulations & 75 \\
\hline Erythromycin & Binary & $542-544$ & $2-20$ & 0.172 & 0.565 & Pharmaceutical formulations and biological fluids & 76 \\
\hline Azithromycin & Binary & $542-544$ & $1-10$ & 0.153 & 0.514 & Pharmaceutical formulations and biological fluids & 76 \\
\hline Clarithromycin & Binary & $542-544$ & $3-30$ & 0.281 & 0.906 & Pharmaceutical formulations and biological fluids & 76 \\
\hline Roxithromycin & binary & $542-544$ & $2-20$ & 0.253 & 0.849 & Pharmaceutical formulations and biological fluids & 76 \\
\hline Ramipril & Ternary & 535 & $20-100$ & - & - & Tablets & 77 \\
\hline Perindopril & Ternary & 535 & $10-60$ & - & - & Tablets & 77 \\
\hline Enalapril & $\begin{array}{l}\text { Ternary } \\
\text { without } \\
\text { surfactant }\end{array}$ & 533.4 & $56-112$ & 1.412 & - & Dosage forms & 78 \\
\hline Enalapril & $\begin{array}{l}\text { Ternary } \\
\text { with } \\
\text { surfactant }\end{array}$ & 558.8 & $20-32$ & 0.587 & - & Dosage forms & 78 \\
\hline Gatifloxacin & Ternary & 552 & $2-10$ & 0.216 & 0.72 & Pharmaceutical formulations & 79 \\
\hline Moxifloxacin & Ternary & 549 & $1-8$ & 0.184 & 0.613 & Pharmaceutical formulations & 79 \\
\hline Memantine $\mathrm{HCl}$ & Binary & 546 & $1-10$ & 0.33 & 0.99 & Tablets & 80 \\
\hline Perindopril erbumine & Ternary & 510 & $10-200$ & 0.49 & 1.48 & Pharmaceutical preparations & 81 \\
\hline Gliclazide & Ternary & 550 & $0.5-4$ & 0.05 & 0.5 & Pharmaceutical formulations and biological fluids & 82 \\
\hline Levofloxacin & Binary & 547 & $2-8$ & 0.1475 & - & $\begin{array}{l}\text { Pure, pharmaceutical tablets and spiked human } \\
\text { urine }\end{array}$ & 83 \\
\hline Norfloxacin & Binary & 547 & $2-8$ & 0.1402 & - & $\begin{array}{l}\text { Pure, pharmaceutical tablets and spiked human } \\
\text { urine }\end{array}$ & 83 \\
\hline Ciprofloxacin & Binary & 547 & $2-8$ & 0.1369 & - & $\begin{array}{l}\text { Pure, pharmaceutical tablets and spiked human } \\
\text { urine }\end{array}$ & 83 \\
\hline Olanzapine & Ternary & 540 & $0-35$ & 0.1501 & 0.4547 & Pure and Dosage Forms & 84 \\
\hline Orphenadrine & Ternary & 540 & $0-55$ & 0.3109 & 0.9422 & Pure and Dosage Forms & 84 \\
\hline Cefixime & Ternary & 550 & $4-28$ & 0.90 & 2.70 & Dosage Forms & 85 \\
\hline Glimepiride & Ternary & 544 & $5-50$ & 1.70 & 5.10 & Dosage Forms & 86 \\
\hline Sparfloxacin & Ternary & 550 & $1.6-16$ & 0.0211 & 0.0704 & Bulk and pharmaceutical preparations & 86 \\
\hline Minocycline & Ternary & 545 & $0-4$ & - & - & Pharmaceutical preparations & 87 \\
\hline Meclizine & Binary & 540 & $5-25$ & 0.76 & 2.29 & Pure and dosage forms & 88 \\
\hline Tolterodine tartrate & Binary & 545 & $1-10$ & 0.10 & 0.31 & Pharmaceutical preparations & 89 \\
\hline Berberine sulphate & Binary & - & - & - & - & Tablets & 90 \\
\hline Solifenacin succinate & Ternary & 545 & $2.5-50$ & 0.116 & 0.351 & Dosage forms & 91 \\
\hline Metoclopramide $\mathrm{HCl}$ & Binary & 543 & $1.01-10.09$ & 0.124 & 0.414 & Dosage forms & 92 \\
\hline Carbinoxamine & Ternary & 538 & $0.75-10$ & - & - & Pharmaceutical formulations & 93 \\
\hline
\end{tabular}

It was found that the binary or ternary complex formed by interaction with eosin alone or eosin and metal measured through spectrofluorimetry offers high sensitivity and selectivity compared to the spectrophotometric analysis. A list of pharmaceuticals reported in table 3 clearly states that spectrofluorimetric methods are more sensitive and have low limit of detection [94-101].

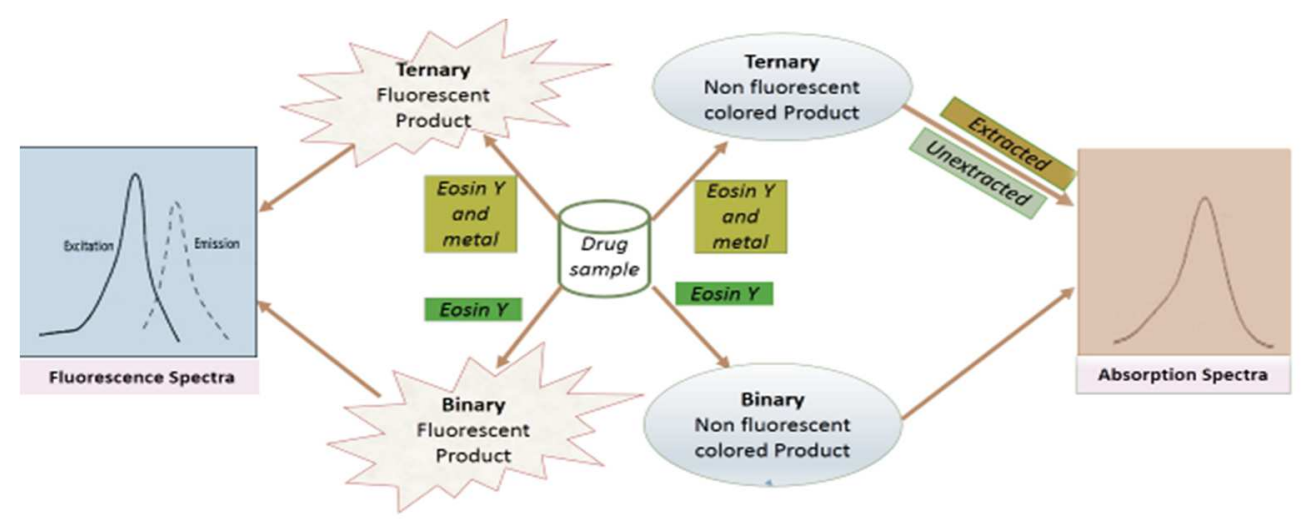

Fig. 4: Schematic diagram for ion association complexation with eosin $Y$ and its analysis 
Table 3: Comparison of spectrophotometric and spectrofluorimetric determination of drugs using eosin $Y$

\begin{tabular}{|c|c|c|c|c|c|c|c|c|c|c|c|c|}
\hline \multirow[t]{2}{*}{ Drug } & \multirow{2}{*}{$\begin{array}{l}\text { Complex } \\
\text { type }\end{array}$} & \multicolumn{4}{|c|}{ Spectrophotometry } & \multicolumn{5}{|c|}{ Spectrofluorimetry } & \multirow[b]{2}{*}{ Application } & \multirow[b]{2}{*}{ Reference } \\
\hline & & $\begin{array}{l}\lambda_{\max } \\
(\mathrm{nm})\end{array}$ & $\begin{array}{l}\text { Range } \\
\mu \mathrm{g} / \mathrm{ml}\end{array}$ & $\begin{array}{l}\text { LOD } \\
\mu \mathrm{g} / \mathrm{ml}\end{array}$ & $\begin{array}{l}\text { LOQ } \\
\mu \mathrm{g} / \mathrm{ml}\end{array}$ & $\begin{array}{l}\lambda_{\text {em }} \\
(\mathrm{nm})\end{array}$ & $\begin{array}{l}\lambda_{\text {ex }} \\
(\mathrm{nm})\end{array}$ & $\begin{array}{l}\text { Range } \\
\mu \mathrm{g} / \mathrm{ml}\end{array}$ & $\begin{array}{l}\text { LOD } \\
\mu \mathrm{g} / \mathrm{ml}\end{array}$ & $\begin{array}{l}\mathrm{LOQ} \\
\mu \mathrm{g} / \mathrm{ml}\end{array}$ & & \\
\hline Dothiepin $\mathrm{HCl}$ & Binary & 540 & $1-10$ & 0.18 & 0.54 & 543 & 304 & $0.3-8$ & 0.11 & 0.34 & $\begin{array}{l}\text { Pure and } \\
\text { dosage forms }\end{array}$ & 94 \\
\hline Hydrochlorothiazide & Ternary & 543 & $8-40$ & 0.046 & 0.138 & 545 & 462 & $\begin{array}{l}0.05- \\
0.25\end{array}$ & 0.013 & 0.039 & Tablets & 95 \\
\hline Indapamide & Ternary & 543 & $8-40$ & 0.041 & 0.123 & 545 & 462 & $\begin{array}{l}0.05- \\
0.25\end{array}$ & 0.014 & 0.044 & Tablets & 95 \\
\hline Xipamaide & Ternary & 543 & $8-32$ & 0.035 & 0.107 & 545 & 462 & $\begin{array}{l}0.05- \\
0.25\end{array}$ & 0.012 & 0.039 & Tablets & 95 \\
\hline Ciprofloxacin & Ternary & 545 & $3-10$ & 0.142 & 0.431 & 540 & 310 & $\begin{array}{l}0.0375- \\
0.070\end{array}$ & 0.0018 & 0.0055 & Pure and tablets & 96 \\
\hline Norfloxacin & Ternary & 545 & $3-10$ & 0.138 & 0.419 & 540 & 310 & $\begin{array}{l}0.025- \\
0.050\end{array}$ & 0.0010 & 0.0033 & Pure and tablets & 96 \\
\hline Ropirnirole & Binary & 546 & $\begin{array}{l}50- \\
500\end{array}$ & - & - & 540 & 350 & $6-150$ & - & - & Dosage forms & 97 \\
\hline Mebeverine $\mathrm{HCl}$ & Binary & 551 & $1-12$ & 0.53 & 1.04 & 540 & 390 & $0.2-3.5$ & 0.11 & 0.21 & $\begin{array}{l}\text { Commercial } \\
\text { Tablets }\end{array}$ & 98 \\
\hline Doxazosin mesylate & Binary & 547 & $2-14$ & 0.393 & 1.191 & 570 & 430 & $1-10$ & 0.0794 & 0.241 & Tablets & 99 \\
\hline Astemizole & Ternary & 547.5 & $\begin{array}{l}4.1- \\
37.6\end{array}$ & - & - & 545 & 462 & $\begin{array}{l}0.94- \\
7.1\end{array}$ & - & - & $\begin{array}{l}\text { Tablets, } \\
\text { Suspension and } \\
\text { capsule }\end{array}$ & 100 \\
\hline Terfenadine & Ternary & 540.7 & $\begin{array}{l}11.8- \\
47.2\end{array}$ & - & - & 545 & 462 & $\begin{array}{l}0.94- \\
7.1\end{array}$ & - & - & $\begin{array}{l}\text { Tablets, } \\
\text { Suspension and } \\
\text { capsule }\end{array}$ & 100 \\
\hline Flunarizine $\mathrm{HCl}$ & Ternary & 547.5 & $\begin{array}{l}2.4- \\
19.1\end{array}$ & - & - & 545 & 462 & $\begin{array}{l}0.94- \\
7.1\end{array}$ & - & - & $\begin{array}{l}\text { Tablets, } \\
\text { Suspension and } \\
\text { capsule }\end{array}$ & 100 \\
\hline Clopidogrel & Binary & 545 & $0.5-9$ & 0.076 & 0.23 & 560 & 499 & $0.2-6$ & 0.0341 & 0.1033 & $\begin{array}{l}\text { Pharmaceutical } \\
\text { preparation }\end{array}$ & 101 \\
\hline
\end{tabular}

\section{CONCLUSION}

As revealed above eosin $\mathrm{Y}$ is one of the important fluorescent ion pairing agent and spectrofluorimetric technique play an important role in the analysis of many pharmaceutical compounds and active current research area as a number of research articles and reviews published every year on this topic [102-108]. Spectrofluorimetry provides an extremely sensitive, selective and simple method for a variety of active pharmaceutical ingredients at a very low detection limits up to pictogram range [109]. A number of pharmaceutical compounds were also analyzed by spectrophotometry that formed a binary and/or ternary colored complex with eosin Y which proves that it is also an important technique of choice even today in pharmaceutical industries. However, the binary and ternary complex formed with eosin Y and analyzed by spectrofluorimetry offered higher sensitivity and selectivity over spectrophotometry.

Chromatographic methods such as high-performance liquid chromatography and gas chromatography were used as a valuable tool for the quantitative analysis of several pharmaceutical ingredients. However, non-chromatographic methods such as spectrofluorimetry and spectrophotometry are still extensively used in research laboratories, hospitals due to low cost, simplicity, portability and ease of operation. Both nonchromatographic methods involved in analyses utilizing the quenching nature of various fluorescent dyes and hence can be used as an alternative to chromatographic methods using eosin $\mathrm{Y}$ as an ion pairing agents for a variety of pharmaceutical compounds. These two methods can be recommended for routine quality control analysis of drugs where time, cost effectiveness and high specificity of analytical techniques are of great importance.

\section{CONFLICT OF INTERESTS}

\section{Declared none}

\section{REFERENCES}

1. Hunger K. Industrial dyes: chemistry, properties, applications. Wiley-VCH, Verlag GmbH and Co. KGa A, Weinhiem; 2003.

2. Seema A, Babulal R. Fluorescence spectrometric study of eosin yellow dye-surfactant interactions. Arab J Chem 2009;2:7-12.
3. Vanzin D, Freitas CF, Pellosi DS, Batistela AR, Machado AEH, Pontes RM. et al. Experimental and computational studies of protolytic and tautomeric equilibria of erythrosin $\mathrm{B}$ and eosin $\mathrm{Y}$ in water/DMSO. RSC Adv 2016;6:110312-28.

4. Nikitina NA, Reshetnyak EA, Svetlova NV, Petrossyan NOM. Protolytic properties of dyes embedded in gelatin films. J Braz Chem Soc 2011;22:857-66.

5. Sabnis RW. Handbook of biological dyes and stains: synthesis and Industrial Applications, John Wiley and Sons, Inc, Hoboken, NJ; 2010. p. 173.

6. Espada J, Valverde P, Stockert JC. Selective fluorescence of eosinophilic structures in grasshopper and mammalian testis stained with haematoxylin-eosin. Histochemistry 1993;99:385-90.

7. Raj G. Photochemistry: In. Photochemical reactions and their kinetics. 5th ed. Meerut: India, Goel publishing house; 2008. p. 125-6.

8. Srivastava V, Singh PP. Eosin Y catalyzed photoredox synthesis: a review. RSC Adv 2017;7:31377-92.

9. Hari DP, Koning B. Synthetic applications of eosin Y in photoredox catalysis. Chem Commun 2014;50:6688-99.

10. Hari DP. Eosin Y catalyzed visible light oxidative C-C and C-P bond formation. Org Lett 2011;13:3852-5.

11. Atul KS, Ruchi C, Lal Dhar SY. Eosin Y catalyzed visible light mediated aerobic photo-oxidative cleavage of the $\mathrm{C}-\mathrm{C}$ double bond of styrenes. Tetrahedron Lett 2015;56:653-6.

12. Waheed AA, Rao KS, Gupta PD. Mechanism of dye binding in the protein assay using eosin dyes. Anal Biochem 2000;287:73-9.

13. Waheed AA, Gupta PD. Application of an eosin B dye method for estimating a wide range of proteins. J Biochem Biophys Methods 1996;33:187-96.

14. Waheed AA, Gupta PD. Estimation of protein using eosin B dye. Anal Biochem 1996;233:249-52.

15. Kristen MM, Michael TM, Lanxuan TD, Chloe EA, Stephan G, Worachart S. et al. Eosin B as a novel antimalarial agent for drug-resistant Plasmodium falciparum. Antimicrob Agents Chemother 2006;50:3132-41.

16. Piergallini $\mathrm{R}$, Loupis $\mathrm{N}$. Teeth whitening composition comprising activating agents, such as Eosin B and erythrosine B. U. S. Pat Appl Publ US 2007128132. Chem Abstr 2007;147:38007.

17. Brumley WC, Farley JW. Determining eosin as a groundwater migration tracer by capillary electrophoresis/laser-induced 
fluorescence using a multi wavelength laser. Electrophoresis 2003;24:2335-9.

18. Chen PL, Liu SP, Liu ZF, Hu XL. Study on the ternary ionassociation complex nanoparticles of palladium (II)-famotidine and halogen-fluorescein dyes by the resonance rayleigh scattering and their application. Sci Sin Chim 2011;41:1059-71.

19. Junbo L, Xiaoli Y, Jinxiang Y, Lina L. Resonance rayleigh scattering and resonance nonlinear scattering methods for the determination of nicardipine hydrochloride using eosin $\mathrm{Y}$ as a probe. RSC Adv 2016;6:25887-93.

20. Jiahong H, Ri Q, Wenpo L, Shaohua X, Zhongrong S, Qiang L, et al. Voltammetric sensor based on eosin Y film modified glassy carbon electrode for simultaneous determination of hydroquinone and catechol. Anal Meth 2014;6:6494-503.

21. Pratim DP, Anurag R, Sumita D, Devi PS. Enhanced stability of $\mathrm{Zn}_{2} \mathrm{SnO}_{4}$ with $\mathrm{N} 719$, N3 and eosin $\mathrm{Y}$ dye molecules for DSSC application. Phys Chem Chem Phys 2016;18:1429-38.

22. Arad A, Mimouni D, Ben-Amitai D, Zeharia A, Mimouni M. Efficacy of topical application of eosin compared with zinc oxide paste and corticosteroid cream for diaper dermatitis. Dermatology 1999;199:319-22.

23. Teresinha L, Philippe D, Alexandre H, Pierre EW. Topical application of eosin to burns produces interference in measurement of serum vancomycin by fluorescence polarization immunoassay. Clin Chem 1997;43:1238-40.

24. Nail A, Ramazan G, Mehmet T. A new atomic absorption-based method development for indirect determination of histamine in fish samples, dairy products, and alcoholic beverages by flame AAS. Anal Meth 2016;25:5142-51.

25. Gao Y, Li Y, Yao L, Li S, Liu J, Zhang H. Catalyst-free activation of peroxides under visible LED light irradiation through photoexcitation pathway. J Hazard Mat 2017;329:272-9.

26. Pan Z, Peng J, Zang X, Lei G, He Y, Liu D. Study on the interaction between three benzimidazole anthelmintics and eosin Y by high performance liquid chromatography associating with resonance light scattering and its application. Anal Bioanal Chem 2016;408:5247-59.

27. Wen T, Li NB, Lu HQ. A turn-on fluorescent sensor for sensitive and selective detection of sodium dodecyl sulfate based on the eosin Y/Polyethyleneimine system. Anal Chem 2013;85:10863-8.

28. Sidney U, Bernard H, Nathan OK, Julius M. Principle of Fluorescence. Fluorescence assay in biology and medicine, A volume in Molecular Biology: An International series of Monographs and text book; 1969. p. 1-41.

29. Pesez M, Bartos J. Colorimetric and fluorimetric analysis of organic compounds and drugs (clinical and biochemical analysis), New York, Marcel Dekker Inc; 1974. p. 803-4.

30. Yoshikazu F, Itsuo M, Kinuko F, Yoshihiro N, Takeshi T. Determination of chlorpromazine, thiamine, lincomycin, ofloxacin and theophylline by ternary complex formation with eosin and palladium (II). Chem Pharm Bull 1987;35:5004-9.

31. Kaur A, Gupta U. Simultaneous spectrophotometric determination of eosin and erythrosine with $\mathrm{Cr}$ (VI) reagent in micellar media using mean centering of ratio spectra. Chem Sci Trans 2012;1:424-30.

32. Chakraborty M, Panda AK. Spectral behaviour of eosin $Y$ in different solvents and aqueous surfactant media. Spectrochim Acta Part A 2011;81:458-65.

33. Kibblewhite J, Drummond GG, Grieser F, Thistlethwaite PJ. Lipoidaleos in and fluorescein derivatives as probes of the electrostatic characteristics of self-assembled surfactant/water interfaces. J Phys Chem 1989;93:7464-73.

34. Lakowicz JR. Principles of fluorescence spectroscopy. In: Lakowicz JR. editors. Quenching of fluorescence. 3rd ed. Springer, Boston, MA; 2006. p. 277-330.

35. Phillips SR, Wilson LJ, Borkman RF. Acrylamide and iodide fluorescence quenching as a structural probe of tryptophan microenvironment in bovine lens crystallins. Curr Eye Res 1986;5:611-9.

36. Punyiczki M, Norman JA, Rosenberg A. Interaction of acrylamide with proteins in the concentration range used for fluorescence quenching studies. Biophys Chem 1993;47:9-19.
37. Lange $\mathrm{R}$, Anzenbacher $\mathrm{P}$, Müller S, Maurin L, Balny C. Interaction of tryptophan residues of cytochrome P45scc with a highly specific fluorescence quencher, a substrate analogue, compared to acrylamide and iodide. Eur J Biochem 1994;226:963-70.

38. Geddes CG. Optical thin film polymeric sensors for the determination of aqueous chloride, bromide and iodide ions at high $\mathrm{pH}$, based on the quenching of fluorescence of two acridinium dyes. Dyes Pigments 2000;45:243-51.

39. Willard DM, Carillo LL, Jung J, Orden AV. CdSe-ZnS quantum dots as resonance energy transfer donors in a model proteinprotein binding assay. Nano Lett 2001;1:469-74.

40. Haas E, Wilchek M, Katzir EK, Steinberg IZ. Distribution of endto-end distances of oligopeptides in solution as estimated by energy transfer. Proc Natl Acad Sci USA 1975;72:1807-11.

41. Lakowicz JR, Gryczynski I, Wiczk W, Laczko G, Franklyn CP, Johnson ML. Conformational distributions of melittin in water/methanol mixtures from frequency-domain measurements of nonradiative energy transfer. Biophys Chem 1990;36:99-115.

42. Chapman ER, Alexander K, Vorherr T, Carafoil E, Storm DR. Fluorescence energy transfer analysis of calmodulin. cntdot. peptide complexes. Biochemistry 1992;31:12819-25.

43. Heyduk T. Measuring protein conformational changes by FRET/IRET. Curr Opin Biotechnol 2002;13:292-6.

44. Hink MA, Bisseling T, Visser AJWG. Imaging protein-protein interactions in living cells. Plant Mol Biol 2002;50:871-83.

45. Parsons $\mathrm{M}$, Vojnovic B, Beg SA. Imaging protein-protein interactions in cell motility using fluorescence resonance energy transfer (FRET). Biochem Soc Trans 2004;32:431-3.

46. El-Rabbat N, Askal HF, Khashaba PY, Attia NN. A validated spectrofluorometric assay for the determination of certain macrolide antibiotics in pharmaceutical formulations and spiked biological fluids. J AOAC Int 2006;89:1276-87.

47. Gillis V, Kubic TA. Spectrofluorometric analysis of illicit drug samples, employing a corrected excitation spectrofluorometer. ISA Trans 1975;14:261-9.

48. Nahata A, Dixit VK. Spectrofluorimetric estimation of scopoletin in Evolvulus alsinoides Linn. and Convolvulus pluricaulis Choisy. Indian J Pharm Sci 2008;70:834-7.

49. Sethiya NK, Nahata A, Dixit VK. Simultaneous spectrofluorimetric determination of scopoletin and mangiferin in a methanolic extract of Canscora decussates Chult. Asian J Trad Med 2008;3:224-9.

50. Gupta NK, Nahata A, Dixit VK. Development of spectrofluorimetric method for the determination of curcumin. Asian J Trad Med 2010;5:12-8.

51. Nahata A, Gupta NK, Dixit VK. A simple and rapid spectrofluorimetric method for the determination of testosterone in biological fluids. Oriental Pharm Exp Med 2011;11:147-51.

52. Belal F, El-Brashy A, El-Enany N, El-Bahay $\mathrm{N}$. Spectrofluorometric determination of olanzapine and fluphenazine hydrochloride in pharmaceutical preparations and human plasma using eosin: application to stability studies. J AOAC Int 2008;91:1309-17.

53. Attia KAM, Mohamad AA, Emara MS. A fluorescence quenching based method for the determination of risperidone in bulk and tablets. Eur J Biomed Pharm Sci 2015;2:17-27.

54. Rahman N, Siddiqui S, Azmi SNH. Spectrofluorimetric method for the determination of doxepin hydrochloride in commercial dosage forms. AAPS PharmSciTech 2009;10:1381-7.

55. Derayea SM, Omar MA, Abdel-Lateef MA, Hassan AI. Development and validation of a new spectrofluorimetric method for the determination of some beta-blockers through fluorescence quenching of eosin Y. Application to content uniformity test. Open Chem 2016;14:258-66.

56. Rahman N, Haque SM. Spectrofluorimetric determination of labetalol hydrochloride in pharmaceutical preparations and urine samples. Int J Biomed Sci 2008;4:140-6.

57. Ibrahim FA, Belal F, El-Brashy A. Fluorometric determination of some aminoquinoline antimalarials using eosin. Microchem J 1989;39:65-70. 
58. Wahba MK, El-Enany N, Belal F. Application of the sternvolmer equation for studying the spectrofluorimetric quenching reaction of eosin with clindamycin hydrochloride in its pure form and pharmaceutical preparations. Anal Methods 2015;7:10445-51.

59. Hammad MA, Omar MA, Eltoukhi WE. Validation of rapid and sensitive spectrofluorimetric assay for determination of four triptans in pure and dosage forms; application to human plasma and content uniformity testing. Pharm Anal Acta 2016;7:1-8.

60. Omar AM, Abdel-Mageed HO, Abdel-Gaber AA, Abdel-Megied MA. Spectrophotometric and spectrofluorimetric determination of certain angiotensin receptor blockers through complex formation. J Pharm Sci Res 2011;3:1499-510.

61. Kaur K, Malik AK. Study on the fluorescence quenching reaction of Amitriptyline and clomipramine hydrochlorides with eosin Y and its analytical application. J Fluoresc 2013;23:533-42.

62. Azmi SNH, Al-Fazari A, Al-Badaei M, Al-Mahrazi R. Utility of eosin $\mathrm{Y}$ as a complexing reagent for the determination of citalopram hydrobromide in commercial dosage forms by fluorescence spectrophotometry. Luminescence 2015;30:1352-9.

63. Derayea S, Omar M, Mohammed B, Ali R. Sensitive spectrofluorimetric protocol for the determination of fluoxetine and paroxetine through binary complex formation with eosin Y. Anal Chem Lett 2016;6:508-17.

64. Walash MI, El-Brashy AM, Metwally MES, Abdelal AA. Fluorimetric determination of ethionamide in pharmaceutical preparations and biological fluids. J Chin Chem Soc 2004;51:1059-64.

65. Attia TZ, Omar MA. Spectrofluorimetric determination of thioridazine and flupentixol in dosage forms; application to content uniformity test. Luminescence 2016;31:1091-7.

66. Arici M, Kilinci E. Validated spectroflurometric method for the determination of sunitinib malate, dye complexation approach for a novel anticancer drug. Acta Pahrm Sci 2010;52:469-85.

67. Ibrahim F, El-Din MKS, Eid MI, Wahba MEK. Validated stabilityindicating spectrofluorimetric methods for the determination of ebastine in pharmaceutical preparations. Chem Cent J 2011;5:1-14.

68. Zakhari NA, Rizk M, Walash MI, Ibrahim F. Fluorimetric determination of certain phenothiazine derivatives using eosin. Anal Lett 1985;18:1405-17.

69. Raghubabu K, Mohan N, Kalyanaramu B, Ramdas C, Rao MN. Development of new visible spectrophotometric determination of tiaprofenic acid in bulk and formulations. Asian J Pharm Clin Res 2013;6:77-9.

70. Sreeja U, Gurupadayya B, Chandan R. Novel spectrophotometric methods for the quantification of desvenlafaxinein pure and pharmaceutical dosage form. Asian J Pharm Clin Res 2015;8:267-70.

71. Lotfy HM, Saleh SS. Recent development in ultraviolet spectrophotometry through the last decade (2006-2016): a review. Int J Pharm Pharm Sci 2016;8:40-56.

72. Walash MI, Belal FF, Eid MI, Mohamed SAE. Spectrophotometric determination of tizanidine and orphenadrine via ion pair complex formation using eosin Y. Chem Cent J 2011;5:1-9.

73. Abd El-Hay SS, El-Mammli MY, Shalaby AA. Determination of clemastine hydrogen fumarate, desloratadine, losartan potassium and mexopril $\mathrm{HCl}$ through binary complex formation with eosin. Arab J Chem 2016;9:S541-7.

74. Derayea SM, Askal HF, Abdelmageed $\mathrm{OH}$, El Hamd MA. Spectrophotometric determination of amlodipine and nicardipine in pharmaceutical formulations via binary complex formation with eosin Y. J Appl Pharm Sci 2012;2:84-9.

75. Dhamra MY, Al-Sabha TN, Al-Ghabsha TS. Spectrophotometric determination of terbutaline sulphate and tetracycline hydrochloride via ion pair complex formation using Eosin Y. Pak J Anal Environ Chem 2014;15:84-92.

76. Walash MI, Rizk MS, Eid MI, Fathy ME. Spectrophotometric determination of four macrolide antibiotics in pharmaceutical formulations and biological fluids via binary complex formation with eosin and spectrophotometry. J AOAC Int 2007;90:1579-87.
77. Abdellatef HE, Ayad MM, Taha EA. Spectrophotometric and atomic absorption spectrometric determination of ramipril and perindopril through ternary complex formation with eosin and Cu (II). J Pharm Biomed Anal 1999;18:1021-7.

78. Ayad MM, Al Shalaby AA, Abdellatef HE. Spectrophotometric and AAS determination of ramipril and enalapril through ternary complex formation. J Pharm Biomed Anal 2002;28:311-21.

79. Gouda AAE, El-Sheikh R, Amin AS. Spectrophotometric determination of some fluoroquinolones antibacterial drugs in pure form and in pharmaceuticals formulations. Anal Chem An Indian J 2008; 7:676-83.

80. Belal TS, Mahrou MS, Daabees HG, Abdel Khalek M, Khami MM. Validated spectrophotometric methods for the determination of memantine hydrochloride in tablets using eosin and 2,4 dinitrofluorobenzene reagents. Res J Pharm Biol Chem Sci 2014;4:1481-93.

81. Rahman N, Rahman H. Quantitative analysis of perindopril erbumine in pharmaceutical preparations by spectrophotometry via ternary complex formation with Zn (II) and eosin and charge transfer complexation with iodine. Spectroscopy 2011;25:123-36.

82. El-Enany N. Spectrophotometric determination of gliclazide in pharmaceuticals and biological fluids through ternary complex formation with eosin and palladium (II). Farmaco 2004;59:63-9.

83. El-Brashy AM, El-Sayed MM, El-Sepai FA. Spectrophotometric determination of some fluoroquinolone antibacterials by binary complex formation with xanthene dyes. Il Farmaco 2004;59:809-17.

84. Deepakumari HN, Revanasiddappa HD. Sensitive spectrophotometric method for the determination of olanzapine and orphenadrine in pure and dosage forms by ternary complex formation with eosin and lead (II). Chem Sci J 2012;70:1-8.

85. Almasri IM, Al-Laham MK. Development and validation of spectrophotometric method for determination of cefixime and glimepiride by ternary complex formation with eosin and $\mathrm{Cu}$ (II). Indo Am J Pharm Res 2014;4:5670-7.

86. El-Didamony AM. Spectrophotometric determination of sparfloxacin in pharmaceutical preparations by ternary complex formation with Pd (II) and eosin. Anal Lett 2007;40:2708-20.

87. Mori I, Fujita Y, Kawabe H, Fujita K, Tanaka T, Kishimoto A. Application of xanthene derivatives in analytical chemistry. Part LVII. Spectrophtometric determination of minocycline using gallium and eosin. Analyst 1986;111:1409-12.

88. Bkhaitan MM, Mirza AZ. Spectrophotometric method for determination of meclizine in pure and dosage forms via ion pair complex formation using eosin Y. Curr Pharm Anal 2016;12:1-6.

89. Belal WF, El-Enany N, Elmansi H. Determination of tolterodine tartrate in pharmaceutical preparations using eosin, application to stability study. Int J Pharma Sci Res 2011;2:2849-55.

90. Zhebentyaev AL, Zhernosek AK. Extraction-spectrophotometric determination of berberine sulphate in tablets using eosin as a reagent. Pharmazie 1996;51:252-5.

91. Burla SVS, Peruri VVS, Chandra BS. Development and validation of spectrophotometric methods for the quantification of solifenacin succinate: application to tablet dosage forms. Int Lett Chem Phys Astron 2013;10:167-82.

92. Naggar AH, Elnasr TAS, Sayed AAS, Kotb A, El Sayed AAY. Determination of metoclopramide hydrochloride in pharmaceutical formulations using three different spectrophotometric methods. Pharm Anal Acta 2017;8:1-9.

93. Ramadan AA, Mandil H. Spectrophotometric determination of carbinoxamine maleate in pharmaceutical formulations by ternary complex formation with $\mathrm{Cu}$ (II) and eosin. Anal Biochem 2006;353:133-7.

94. Walash MI, Belal F, El-Enany N, Elmansi H. Spectrophotometric and spectrofluorimetric methods for the determination of dothiepin hydrochloride in its pure and dosage forms using eosin. Int J Biomed Sci 2010;6:327-34. 
95. Omar MA. Spectrophotometric and spectrofluorimetric determination of certain diuretics through ternary complex formation with eosin and lead (II). J Fluorescence 2010;20:275-81.

96. El-Walily AFM, Belal F, Bakry RS. Spectrophotometric and spectrofluorimetric determination of ciprofloxacin and norfloxacin by ternary complex formation with eosin and palladium (II). J Pharm Biomed Anal 1996;14:561-9.

97. Souri E, Torabad M, Tehrani MB. Spectrophotometric and spectrofluorimetric methods for the determination of ropinirole content in pharmaceutical dosage forms. J Chin Pharm Sci 2016;25:876-81.

98. Deraeya SMS. An application of eosin Y for the selective spectrophotometric and spectrofluorimetric determination of mebeverine hydrochloride. Anal Meth 2014;6:2270-5.

99. Ammar RA, El-Brashy AM, Al-Sahly TN. Spectrophotometric and spectrofluorometric determination of doxazosin mesylate in tablets via eosin Y. Asian J Chem 2014;26:57-62.

100. Kelania K, Bebawyb LI, Abdel-Fattah L. Determination of astemizole, terfenadine and flunarizine hydrochloride by ternary complex formation with eosin and lead (II). J Pharm Biomed Anal 1999;18:985-92.

101. El-Din MS, Ibrahim F, Shalan S, El-Aziz H. New validated spectrofluorimetric and spectrophotometric methods for the determination of clopidogrel in pharmaceutical preparations using eosin. Int J Pharm 2016;6:53-63.

102. Elokely KM, Eldawy MA, Elkersh MA, El-Moselhy TF. Fluorescence spectrometric determination of drugs containing $\alpha$-methylene sulfone/sulfonamide functional groups using $\mathrm{N}^{1}$-methylnicotinamide chloride as a fluorogenic agent. Int J Anal Chem 2011;1-9. http://dx.doi.org/ $10.1155 / 2011 / 840178$

103. Darwish IA, Khedr AS, Askal HF, Mahmoud RM. Simple fluorimetric method for determination of certain antiviral drugs via their oxidation with cerium (IV). Il Farmaco 2005;60:555-62.

104. Mazzarino L, Bellettini IC, Minatti E, Lemos-Senna E. Development and validation of a fluorimetric method to determine curcumin in lipid and polymeric nanocapsule suspensions. Braz J Pharm Sci 2010;46:219-26.

105. El-Shaboury SR, Hussein SA, Mohamed NA, El-Sutohy MM. Spectrofluorimetric method for determination of some angiotensin II receptor antagonists. J Pharm Anal 2012;2:12-8.

106. Gouda AA, El-Sayed MIK, Amin AS, El Sheikh R. Spectrophotometric and spectrofluorometric methods for the determination of non-steroidal anti-inflammatory drugs: a review. Arab J Chem 2013;6:145-63.

107. Omar MA, Abdelmageed OH, Derayea SM, Uno T, Atia TZ. Spectrofluorimetric determination of certain antidepressant drugs in human plasma. J Anal Sci Tech 2013;4:1-10.

108. Bonfilio R. Recent applications of analytical techniques for quantitative pharmaceutical analysis: a review. Wseas Transactions Bio Biomed 2010; 7:316-38.

109. Kaur K, Singh B, Malik AK. Chemiluminescence and spectrofluorimetric methods for determination of fluoroquinolones: a review. Anal Lett 2011;44:1602-39. 